OPEN ACCESS

Edited by: Nicola J. Smith

Victor Chang Cardiac Research Institute, Australia

Reviewed by:

Natasha L. Grimsey, University of Auckland, New Zealand Simon R. Foster,

University of Copenhagen, Australia

*Correspondence:

Heike Biebermann heike.biebermann@charite.de

Specialty section: This article was submitted to Molecular and Structural Endocrinology,

a section of the journal Frontiers in Endocrinology

Received: 12 May 2016 Accepted: 26 July 2016 Published: 08 August 2016

Citation: Müller A, Niederstadt L, Jonas $W$, Yi C-X, Meyer F, Wiedmer P, Fischer J, Grötzinger C, Schürmann A, Tschöp M, Kleinau G, Grüters A, Krude H and Biebermann H (2016) Ring Finger Protein 11 Inhibits Melanocortin 3 and 4 Receptor Signaling.

Front. Endocrinol. 7:109. doi: 10.3389/fendo.2016.00109

\section{Ring Finger Protein 11 Inhibits Melanocortin 3 and 4 Receptor Signaling}

\begin{abstract}
Anne Müller ${ }^{1}$, Lars Niederstadt' ${ }^{2}$, Wenke Jonas ${ }^{3,4}$, Chun-Xia Yi ${ }^{5}$, Franziska Meyer Petra Wiedmer ${ }^{3}$, Jana Fischer ${ }^{1}$, Carsten Grötzinger ${ }^{2}$, Annette Schürmann ${ }^{3,4}$, Matthias Tschöp ${ }^{7,8}$, Gunnar Kleinau ${ }^{1}$, Annette Grüters ${ }^{1}$, Heiko Krude ${ }^{1}$ and Heike Biebermann ${ }^{1 *}$

${ }^{1}$ Institut für Experimentelle Pädiatrische Endokrinologie, Charité-Universitätsmedizin Berlin, Berlin, Germany, ${ }^{2}$ Tumor Targeting Laboratory, Department of Hepatology and Gastroenterology, Molecular Cancer Research Center (MKFZ), Charité-Universitätsmedizin Berlin, Berlin, Germany, ${ }^{3}$ Department of Experimental Diabetology, German Institute of Human Nutrition Potsdam-Rehbruecke (DIfE), Nuthetal, Germany, ${ }^{4}$ German Center of Diabetes Research, Neuherberg, Germany, ${ }^{5}$ Department of Endocrinology and Metabolism, Academic Medical Center (AMC), University of Amsterdam, Amsterdam, Netherlands, ${ }^{6}$ Institut für Experimentelle Endokrinologie, Charité-Universitätsmedizin Berlin, Berlin, Germany, ${ }^{7}$ Institute for Diabetes and Obesity, Helmholtz Zentrum München, Germany, Deutsches Forschungszentrum für Gesundheit und Umwelt $(\mathrm{GmbH})$, Neuherberg, Germany, ${ }^{8}$ Technische Universität München, München, Germany
\end{abstract}

Intact melanocortin signaling via the G protein-coupled receptors (GPCRs), melanocortin receptor $4(\mathrm{MC} 4 \mathrm{R})$, and melanocortin receptor $3(\mathrm{MC} 3 \mathrm{R})$ is crucial for body weight maintenance. So far, no connection between melanocortin signaling and hypothalamic inflammation has been reported. Using a bimolecular fluorescence complementation library screen, we identified a new interaction partner for these receptors, ring finger protein 11 (RNF11). RNF11 participates in the constitution of the A20 complex that is involved in reduction of tumor necrosis factor $\alpha(T N F \alpha)$-induced NFkB signaling, an important pathway in hypothalamic inflammation. Mice treated with high-fat diet (HFD) for 3 days demonstrated a trend toward an increase in hypothalamic Rnf11 expression, as shown for other inflammatory markers under HFD. Furthermore, Gs-mediated signaling of MC3/4R was demonstrated to be strongly reduced to $20-40 \%$ by co-expression of RNF11 despite unchanged total receptor expression. Cell surface expression was not affected for MC3R but resulted in a significant reduction of MC4R to $61 \%$ by co-expression with RNF11. Mechanisms linking HFD, inflammation, and metabolism remain partially understood. In this study, a new axis between signaling of specific body weight regulating GPCRs and factors involved in hypothalamic inflammation is suggested.

Keywords: G protein coupled receptor, protein complementation assay, protein network, weight regulation, inflammation

\section{INTRODUCTION}

Obesity and its associated comorbidities, such as type 2 diabetes or cardiovascular diseases, represent critical global health issues. Increasing caloric intake and/or reduced energy expenditure leads to excessive energy storage resulting in adipose accumulation, coupled to chronic low-grade inflammation (1-3) and weight gain. Numerous high-fat diet (HFD) analyses have revealed that inflammation not only occurs in peripheral tissue but also results in a non-classical form of 
inflammation in the hypothalamus, particularly in the arcuate (ARC) and paraventricular nucleus (PVN) $(1,3)$. Hypothalamic inflammatory changes have been detected within a short period of 3 days HFD $(1,4)$. In addition to microglia invasion, gene expression analyses revealed increased hypothalamic levels of inflammatory markers such as interleukin 6 (Il6), tumor necrosis factor $\alpha$ (Tnf $\alpha)$, suppressor of cytokine signaling 3 (Socs3), and inhibitor of nuclear factor kappa-B kinase subunit beta or epsilon $(I k b k b / I k b k e)(1,4)$. The known functions of leptin and insulin receptors have been reported to be affected by HFD-induced hypothalamic inflammation. Hypothalamic leptin resistance has already been shown to develop very early in the first 1-3 days of HFD (5). Upregulation of Socs3 and various inflammatory pathways are presumed to be contributors to leptin insensitivity $(5,6)$. Continuous HFD, inflammation, and manifesting leptin resistance lead to peripheral and hypothalamic insulin insensitivity (7).

Aside from leptin and insulin receptors, the $G$ protein-coupled receptors (GPCR) and melanocortin 3 and 4 receptor (MC3/4R) are important regulators of body weight and energy consumption, as part of the hypothalamic leptin-melanocortin pathway (8).

Regarding the leptin-melanocortin axis, PVN expressed MC4R is the most prominent MCR in weight regulation $(9,10)$. MC4R knockout or loss-of-function variants in humans result in hyperphagia and early onset obesity (11-13). Furthermore, MC3R is expressed in the ARC on POMC neurons and agoutirelated peptide (AgRP) neurons. MC3R knockout mice develop increased fat mass, hyperleptinemia, or hyperinsulinemia $(14,15)$. $\mathrm{MC} 3 / 4 \mathrm{R}$ activity is primarily controlled by $\mathrm{MSH}$, processed from POMC in anorexigenic POMC neurons and by AgRP, which is expressed in orexigenic AgRP neurons $(10,16,17)$.

To further investigate the role of MC3R and MC4R, we applied a screening system that identified ring finger protein 11 (RNF11) as a potential hypothalamic melanocortin receptor interaction partner. RNF11 is a highly conserved E3 ligase, with different physiological functions (18) and operates as a compound of the A20 complex. The A20 complex is an ubiquitin-editing enzyme that plays a critical role in the termination of TNF $\alpha$-induced canonical NFKB signaling pathway $(19,20)$.

In the present study, we tried to confirm the MCR/RNF11 interaction and analyzed the impact of RNF11 on MCR function in particular with regard to the early developed hypothalamic inflammatory changes during HFD.

\section{MATERIALS AND METHODS}

\section{Vector Construction}

$M c 3 r, M c 4 r$, Ghsr, Gpr83, and Rnf11 were amplified from murine hypothalamic cDNA. $M C 3 R$ was amplified from human genomic DNA and GHSR from human cDNA (UMR cDNA Resource Center, Rolla, MO, USA). The pcDps [NHA-Chrm3], origin of all rCHRM3 (rat cholinergic receptor, muscarinic 3) constructs, was kindly provided by Torsten Schöneberg (University of Leipzig, Germany). The origin of YFP-fragment constructs was pcDNA3 (MCFD2-YFP1/YFP2) plasmids, which were kindly provided by Hans-Peter Hauri (University of Basel, Switzerland) (21). For direct interaction analysis of two known proteins in $\mathrm{YFP}-\mathrm{BiFc}$,
MCFD2 was replaced by the here relevant full-length genes (without stop codons). The pECFP-N1 (Mc3r-YFP1) (kanamycin resistance), required for the $\mathrm{YFP}-\mathrm{BiFc}$ screening, was created by replacement of ECFP with Mc3r-YFP1. pECFP-N1 was purchased from Invitrogen (Darmstadt, Germany). The normalized murine hypothalamic cDNA library for screening was produced from wild-type hypothalami and inserted into pcDNA3-YFP2 (ampicillin resistance) by Eurofins MWG Operon (Ebersberg, Germany). The hemagglutinin tag was inserted at the amino terminus (NHA) and the FLAG tag at the carboxyl terminus. Reporter constructs pGL4.30 (luc2P/NFAT-RE/Hygro) and pGL4.33 (luc2P/SRE/Hygro) were purchased from Promega (Mannheim, Germany). The pGL4.34 (luc2P/NFkB-RE/Hygro) for detection of NFKB-activity was kindly provided by Vera Knaeuper (Cardiff University, Cardiff, United Kingdom).

\section{Cell Culture and Transfection}

HEK293 and COS-7 cells were cultured as described elsewhere (22). For bimolecular fluorescence complementation (BiFc) approaches, HEK293 cells were seeded in 6 -cm dishes $\left(8.5 \times 10^{5}\right.$ cells/dish) and transfected with 1.9 $\mu$ g DNA: $5.9 \mu$ Metafectene $^{\mathrm{TM}} /$ dish (Biontex, Martinsried, Germany). For cAMP accumulation and cell viability tests, COS-7 cells were seeded into 96-well plates $\left(0.9 \times 10^{4}\right.$ cells/well $)$. For measurement of NFAT, SRE and NFkB activity via reporter gene assays, HEK293 cells were seeded in 96-well plates $\left(1.5 \times 10^{4}\right.$ cells/well $)$ coated with poly-L-lysine (Biochrom, Berlin, Germany). Cell viability of HEK293 cells was also examined in 96-well plates. Transfection in 96-well plates was performed with $41.7 \mathrm{ng}$ plasmid DNA/well and $0.5 \mu \mathrm{l}$ Metafectene $e^{\mathrm{TM}} /$ well. For reporter gene assays, equal amounts of the appropriate reporter construct containing the firefly luciferase gene was co-transfected. For cell surface expression studies, COS-7 cells were seeded into 48 -well plates $\left(3.8 \times 10^{5}\right.$ cells/well $)$ and transfected with $167 \mathrm{ng}$ DNA and $1 \mu \mathrm{l}$ Metafectene $\mathrm{T}^{\mathrm{TM}} /$ well (22). Total expression studies via sandwich ELISA were performed with COS-7 cells seeded in 6-cm dishes $\left(6.5 \times 10^{5}\right.$ cells/ dish) and transfected with $3 \mu \mathrm{g}$ DNA and $8 \mu$ l Metafecten $e^{\mathrm{TM}} /$ dish. All transfections were performed 1 day after seeding.

Cell lines that were chosen were well established in respective assays. YFP-BiFc analysis was performed in HEK293 cells due to optimal transfection results in combination with well detectable fluorescence signals. In addition to HEK293 cells, more robust COS-7 cells were used for wash intensive ELISA systems in expression studies. In regards to both cell lines, functional receptor studies were performed in HEK293 and COS-7 cells.

\section{Interaction Partner Screening and Association Studies via YFP-Based Bimolecular Fluorescence Complementation Assay}

For screening, pECFP-N1 (Mc3r-YFP1) and pcDNA3 (hypothalamic cDNA library-YFP2) were co-transfected. Two days after transfection, cells were harvested and analyzed for fluorescence using ArialI SORP and the FACSDiva v6 program (Becton Dickinson, Heidelberg, Germany) at the Berlin-Brandenburg Center for Regenerative Therapies (BCRT, Charité Berlin). The 
cell sorter was adjusted with an interaction positive [MC3RYFP1 + GHSR-YFP2 (23)] and negative control [rCHRM3YFP1 + GHSR-YFP2 (24)]. Plasmid DNA from YFP-positive cells was purified using QIAmp DNA Blood Mini Kit (Qiagen, Hilden, Germany) and introduced into E. coli cells. Clones carrying library constructs were selected via antibiotic resistance, analyzed by sequencing, and evaluated via NCBI-BLAST.

Direct interaction or association between two defined proteins was analyzed in YFP-BiFc experiments using the full length genes as previously described (24). YFP-fluorescent cells were measured using the FACS Canto II at the BCRT and were analyzed with FlowJo 8.8.6 (Tree Star Inc., Ashland, OR, USA).

\section{Measurement of Signaling Pathways}

Signaling pathways were analyzed $48 \mathrm{~h}$ after transfection. Intracellular cAMP accumulation for the determination of Gs activation was analyzed using the AlphaLISA technology (PerkinElmer, Rodgau, Germany). Human $\alpha$-MSH was purchased from Sigma-Aldrich (Taufkirchen, Germany). Stimulation was performed for $45 \mathrm{~min}$. Cell lysis $(50 \mu \mathrm{l} /$ well lysis buffer $)$ and cAMP measurement were conducted as described elsewhere (22) according to the manufacturer's protocol (PerkinElmer).

NFAT, SRE, and NFKB activity were determined in luciferase reporter gene assays. Stimulations with $\alpha-\mathrm{MSH}$, TNF $\alpha$, and Ghrelin (Sigma-Aldrich) were performed for $6 \mathrm{~h}$ to allow appropriate reporter expression and according to the manufacturer's instructions (Promega). Subsequently, cell lysis was performed with $50 \mu \mathrm{l} /$ well of $1 \times$ Passive Lysis Buffer (Promega). Pathway activities were determined by luciferase activity according to the manufacturer's protocol (Promega).

\section{Cell Surface and Total Expression Studies}

Cell surface and total expression studies were performed in COS-7 cells $48 \mathrm{~h}$ after transfection using ELISA systems as previously described $(22,24)$. To investigate cell surface expression, HA-tagged receptors were required. For detection of the HA-tag, cells were washed, fixed with paraformaldehyde, and probed with a biotin-labeled anti-HA antibody (Roche Applied Science, Mannheim, Germany). Bound biotin anti-HA antibody was detected by peroxidase-labeled streptavidin (BioLegend, London, UK) in a substrate/chromogen reaction as previously described (25).

Total expression was measured using HA and FLAG doubletagged receptors. Cells were solubilized at $4^{\circ} \mathrm{C}[10 \mathrm{mM}$ Tris/ $\mathrm{HCl}$, pH 7.4, $150 \mathrm{mM} \mathrm{NaCl}, 1 \mathrm{mM}$ EDTA, $1 \mathrm{mM}$ DTT, 1\% sodium deoxycholat, 1\% NP-40 and 0.2 mM PMSF (22)]. Lysates were incubated in anti-FLAG antibody (Sigma-Aldrich)-coated 96-well plates for $2 \mathrm{~h}$. The HA epitope was detected as specified above. Total protein concentration of lysates was measured using a bicinchoninic acid (BCA)-based protein assay (Thermo Scientific, Bonn, Germany).

\section{Cell Viability Tests}

Cell viability was measured using the CellTiter $96^{\circledR}$ Aqueous One Solution Cell Proliferation Assay (Promega) according to the manufacturer's instructions $\left(10 \mu \mathrm{l} 96^{\circledR}\right.$ solution/50 $\mu \mathrm{l}$ medium $)$. Viability tests were performed $48 \mathrm{~h}$ after transfection.

\section{Animals and cDNA Synthesis}

All procedures were approved by the Animal Care and Use Committee at the State Ministry of Rural Development, Environment and Consumer Protection of Brandenburg, Germany, in accordance with the German Animal Welfare Act (V3-2347-04-2013).

Hypothalami from ad libitum standard chow (S, $3.3 \mathrm{kcal} \%$ fat; Ssniff GmbH, Soest, Germany) and high-fat (HF, $60 \mathrm{kcal} \%$ fat; Research Diets Inc., New Brunswick, NJ, USA) diet-fed male mice (C57BL/6J) were sampled at an age of 6-6.5 weeks. HFD was administered for 3 days before salvation. RNA extractions were performed using $1 \mathrm{ml}$ TRIzol $^{\circledR}$ reagent (Sigma-Aldrich) per hypothalamus according to the manufacturer's instructions. Following DNaseI (New England Biolabs, Frankfurt am Main, Germany) digestion and before reverse transcription, the secondary structure of RNA was minimized by incubation at $60^{\circ} \mathrm{C}$ for $10 \mathrm{~min}$. Synthesis of cDNA was performed using $400 \mathrm{ng}$ RNA with $0.5 \mathrm{mM}$ dNTP-Mix (Qiagen), $25 \mathrm{ng} / \mu$ l Oligo(dT)15-Primer, and M-MLV Reverse Transcriptase under use of RNasin ${ }^{\circledR}$ Plus RNase Inhibitor (Promega).

\section{Quantitative Real-time PCR}

Quantitative real-time PCR was performed using the SsoFast ${ }^{\mathrm{TM}}$ EvaGreen ${ }^{\circledR}$ Supermix (Bio-Rad, Hercules, CA, USA). Primers specific for six reference genes were analyzed for the compared murine samples: ribosomal protein, large, $P 0$ (Rplp0), $18 \mathrm{~S}$ ribosomal RNA (Rn18S), beta-actin (Actb), polymerase (RNA) II (DNA directed) polypeptide A (Polr2a), TATA box binding protein (Tbp), and hypoxanthine guanine phosphoribosyl transferase (Hprt). The reference gene $A c t b$ was chosen in this experiment due to its minimal variation across experimental groups (analyzed by geNorm of the qBase software, Zulte, Belgium). Quantitative PCR data were analyzed using the comparative $\mathrm{Ct}$ method. $\mathrm{Ct}$ is the quantification cycle number at which the light outcome of qPCR is first recorded above the background signals. The difference of the average $\mathrm{Ct}$ value for the gene of interest and the average $\mathrm{Ct}$ value for the reference gene represents $\Delta \mathrm{Ct}$. To receive the relative quantitation of gene expression, the arithmetic formula $2^{-\Delta \mathrm{Ct}}$ was used (Applied Biosystems' User Bulletin 2, Foster City, CA, USA).

\section{Statistical Analyses}

All data were checked for normal distribution and expressed as mean \pm or + SEM. Statistical analyses were performed using the statistical tools implemented in Graph Pad Prism, version 6 (GraphPad Software, San Diego, CA, USA).

\section{RESULTS}

\section{RNF11 as Screening Hit for a Potential MC3R Interaction or Association}

In the initial screening via YFP-based bimolecular fluorescence complementation (YFP-BiFc), we investigated potential new murine hypothalamic MC3R interaction partners. The MC3R was chosen due to its known and particularly wellcharacterized capability to interact in a functional relevant manner with several other proteins such as receptors and 
accessory proteins $(23,26,27)$. The initial sorting process was stopped after $30 \mathrm{~min}$. During this time, 3,900 YFP fluorescent cells were collected. Subsequent selection for library constructs resulted in 239 bacterial clones. Sequence analysis, in which false positive hits were sorted out (e.g., cDNA fragment not in frame with YFP fragment, cDNA fragment consisting of non-coding DNA region), revealed 16 potential hypothalamic interaction partners for the murine MC3R. The detected potential interaction partners were proteins that are typically found in multiple protein complexes or involved in trafficking processes, cell metabolism, protein degradation, cell-cell communication, and cellular nutrient sensing (Table S1 in Supplementary Material). In the present study, we focused on the interplay with RNF11. RNF11 plays a crucial role in reducing $\mathrm{NF \kappa B}$ signaling and, therefore, in the counterregulation of inflammation. RNF11 represents an important component

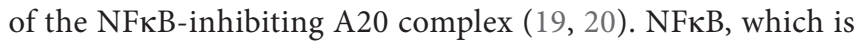
activated by $\mathrm{TNF} \alpha$, has been shown to result in an upregulation of A20 expression and in a higher interactivity of RNF11, assembling the A20 complex and protecting cells from constant NFкB signaling (20). Known hypothalamic expression, present structural protein-binding motifs $(28,29)$, and previously published interaction findings $(19,20)$ give rise to the possibility that RNF11 represents a potential and important interaction partner for neuronal GPCRs of weight regulation.

In addition to MC3R, we also concentrated on the most important GPCR in hypothalamic weight regulation, the MC4R. MC3R and MC4R share a high sequence similarity. Therefore, further analyses focused on both melanocortin receptors.

\section{RNF11 Interacts with MC3R and MC4R}

To confirm MC3R/RNF11 interaction and further examine RNF11/MC4R interplay, YFP-BiFc studies with full-length genes were performed (Figure 1). The co-expression of GHSR and a non-interactive protein (NIP) represented by the rat cholinergic receptor, muscarinic 3 [rCHRM3 (24), Figure 1, first white column] served as interaction negative and the MC3R/GHSR heterodimer as interaction positive controls for GPCRs [(23), Figure 1, black column]. Furthermore, previous studies of YFP-BiFc have shown that GPCRs do not randomly aggregate with any other co-expressed and overexpressed proteins in this assay system (24). Interaction negative controls for $\mathrm{MC} 3 / 4 \mathrm{R}$ (co-expression with NIP, rCHRM3) have been previously shown $(24,30)$, and similar results were reported as the negative control in the present study. To establish RNF11 interaction controls, homodimerization of the soluble protein was demonstrated (Figure 1, dark gray column) in addition to non-interaction with rCHRM3 (Figure 1, third column). Interaction of RNF11 with $\mathrm{MC} 3 / 4 \mathrm{R}$ was confirmed via full-length YFP-BiFc (Figure 1, light gray columns).

In BiFc studies, fluorescence analysis does not provide quantitative information of intracellular interaction as it is usually irreversible. Equal expression of transfected YFP1 and YFP2 tagged proteins was assumed but not verified. Furthermore, due to the detection of interactions within a distance of several nanometers by $\mathrm{BiFc}$, interaction partners that act imbedded in complexes and direct protein-protein interactions can be identified using BiFc.

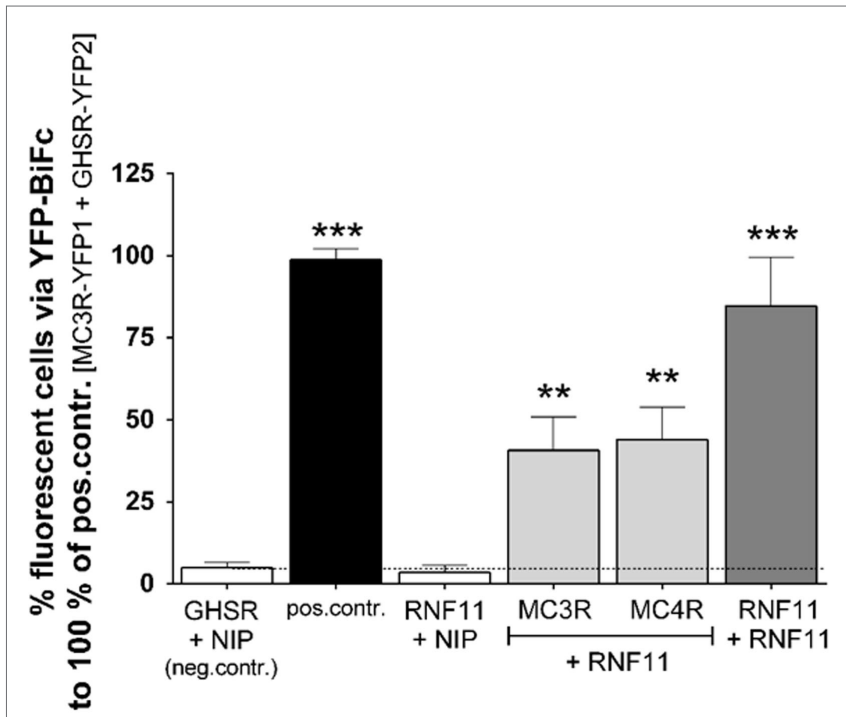

FIGURE 1 | RNF11 interacts with MC3/4R. Interaction studies via YFP-based protein complementation assay (YFP-BiFc). Positive control, human MC3R/GHSR heterodimer (23); negative control, GHSR/noninteracting protein (NIP, rCHRM3) co-expression (24). No YFP1/YFP2 tags are displayed due to the combination of values from vice versa assays (e.g., first gray column: values from RNF11-YFP1 + MC3R-YFP2 and values from RNF11-YFP2 + MC3R-YFP1 experiments). Fifty thousand cells were analyzed on fluorescence per sample and experiment. Data were assessed from a minimum of three independent experiments, each performed in triplicates, and calculated to the control. Values represent the mean + SEM. Significance to negative control ${ }^{* \star *} p \leq 0.001$ (one-way ANOVA, Dunnett's test).

In a sandwich ELISA system including destructive cell lysis and several washing steps, RNF11 interactions were not detected. Therefore, we cannot exclude that further cellular proteins are involved in, or even mediate, the MC3R and MC4R interaction with RNF11. For simplification purposes, the MCR/RNF11 interplay will be termed here as interaction.

\section{MC3/4R Signaling Capacity Is Inhibited by RNF11}

Alpha-MSH-induced cAMP accumulation (Gs signaling) through MC3R (Figure 2A) and MC4R activation (Figure 2B) in co-expression with the NIP $[\operatorname{rCHRM} 3(24,30)]$ resulted in typical concentration response curves with a stimulation maxima of 160 to 200-fold cAMP accumulation compared with the basal empty vector. EC50 values of MCR/NIP co-expressions were measured at 5-8 nM $\alpha$-MSH (Figures 2A,B). Co-expression with RNF11 led to a reduction to $44 \%$ of MC3R signaling and $19 \%$ of MC4R signaling in Gs in response to $100 \mathrm{nM} \alpha-\mathrm{MSH}$ without an effect on the EC50 value (Figures 2A,B). In addition to the main Gs pathway, ligand-induced activation of MC4R has been reported to modify the activity of extracellular regulated kinases $[(31,32)$ ERK 1/2 signaling], AMP-activated kinases (33), c-jun kinases (34), protein kinases $C$ (35), and phosphatidylinositol-3-kinases $[(31,36)$ IP3 formation]. To investigate whether RNF11 specifically inhibits Gs signaling of MCRs, two additional pathways were 

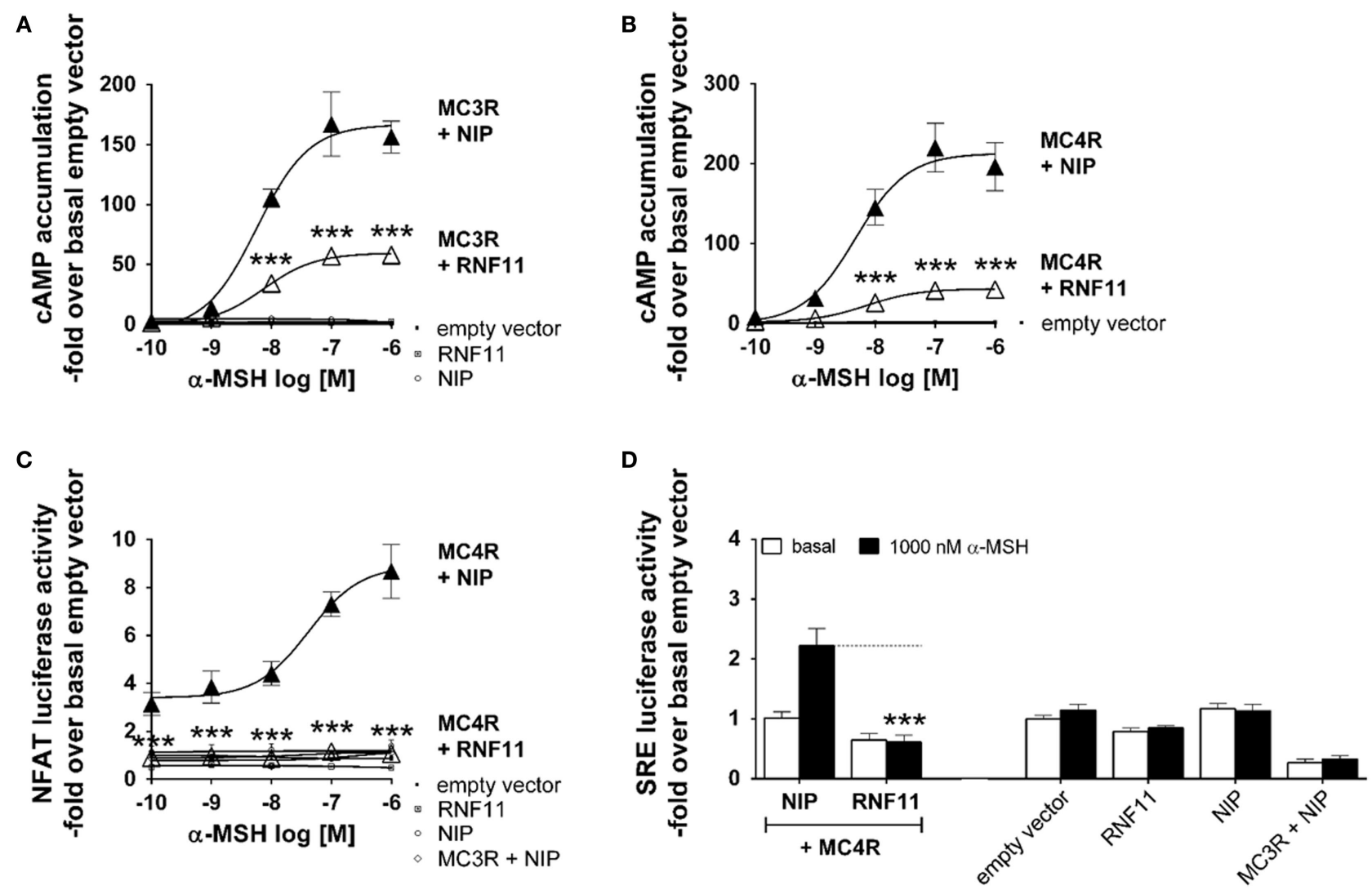

FIGURE 2 | RNF11 inhibits MC3/4R signaling. Functional studies of MC3R and MC4R in cAMP accumulation (A,B), NFAT (C), and SRE-controlled luciferase activity (D). Concentration response curves to $\alpha-\mathrm{MSH}$ (A-C). MC3/4R co-expressions with a non-interactive protein [NIP, rCHRM3 (30); black filled triangles] are opposed to the GPCR/RNF11 interplay (unfilled triangles). In (D) MC4R, co-expression with NIP (first column pair) is opposed to the MC4R/RNF11 interaction (second column pair) in the absence (basal) or presence of $\alpha-\mathrm{MSH}$. For assay control, single gene or plasmid transfections of the empty vector, RNF11, and NIP were performed. To ensure equal amounts of plasmid DNA in each transfection, single gene approaches were filled with empty vector. Data were assessed from a minimum of three independent experiments, each performed in triplicates. Values represent mean \pm SEM. Significance was determined as stimulated MCRs + NIP to stimulated MCRs + RNF11. ${ }^{* \star *} p \leq 0.001$ (two-way ANOVA for factor RNF11 and $\alpha-M S H$, Sidak test due to extreme low SDs in co-expressions with RNF11 compared with MCRs/NIP).

analyzed (Figures 2C,D). The activity of the transcription factor NFAT and therefore NFAT-driven luciferase activity (Figure 2C) occurs downstream of IP3 formation. SRE-driven luciferase activity (Figure 2D) suggests an upstream MAPK/ERK 1/2 activity. The murine MC4R in co-expression with NIP showed a 3.1-fold basal NFAT luciferase activity compared with the empty vector control (Figure 2C). Furthermore, stimulation with $\alpha-\mathrm{MSH}$ also resulted in a distinct concentration response curve with a maximum 8.6-fold of NFAT luciferase activity compared with the basal empty vector with an EC50 value of $70 \mathrm{nM} \alpha-\mathrm{MSH}$ (Figure 2C). Co-expression of RNF11 resulted in a complete loss of MC4R-mediated NFAT-driven luciferase activity (Figure 2C). In the SRE pathway (Figure 2D), no basal activity of MC4R/NIP co-expression was detected. Stimulation with $1000 \mathrm{nM} \alpha-\mathrm{MSH}$ led to a slight 2.2-fold increase in SRE luciferase activity compared with the basal empty vector, supporting previous data that ERK 1/2 mainly results from Gq/11 (IP3 formation) (31) (Figure 2D). Consistently, also for SRE signaling, RNF11 co-expression diminished MC4R-mediated signaling in total as reported for NFAT activity (Figures 2C,D). For the murine MC3R, neither basal nor $\alpha$-MSH stimulated NFAT or SRE luciferase activity was detected (Figures 2C,D). The expression of the empty vector, RNF11 and NIP (rCHRM3) alone (served as controls), did not show basal activity or stimulation by $\alpha-\mathrm{MSH}$ in the investigated signaling pathways (Figure 2).

\section{MC3/4R Expression Profiles Are Differently Modulated by RNF11}

Cell surface and total expression studies were performed (Figures 3A,B). Using this approach, we aimed to investigate whether the observed functional influence on $\mathrm{MC} / 4 \mathrm{R}$ in the presence of RNF11 (Figure 2) was due to aberrant receptor expression. Co-transfection with NIP (Figures 3A,B, white columns) was compared with MC3R/RNF11 or MC4R/RNF11 co-transfections (Figures 3A,B, black columns). Cell surface expression of MC3R was not significantly reduced by RNF11 (Figure 3A). However, the cell surface presence of MC4R was 

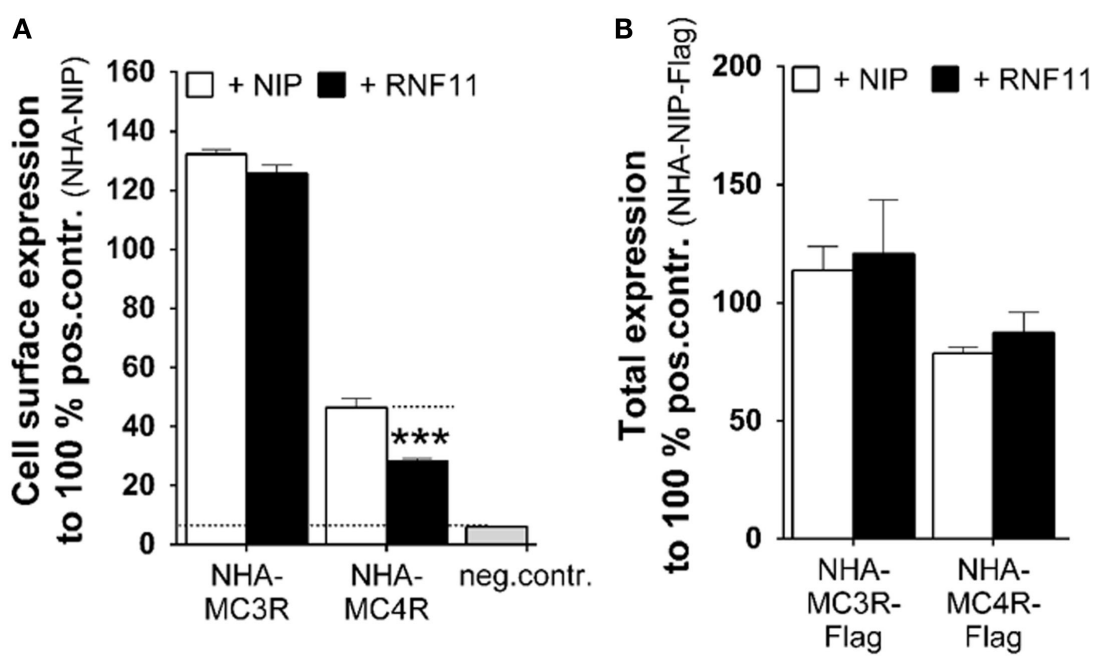

FIGURE 3 | Expression of MC3/4R in the presence of RNF11 is differentially influenced. (A) Cell surface expression and (B) total expression of MC3/4R was analyzed in ELISA systems. The negative control in both expression studies was untagged rCHRM3 (NIP). Positive controls were NHA-rCHRM3 [NHA-NIP (A)] and NHA-rCHRM3-FLAG [NHA-NIP-Flag (B)]. MC3/4R co-expression with NIP (white columns) is opposed to the GPCR/RNF11 interplay (black columns). In cell surface expression studies, GPCRs were detected via the N-terminal HA. In total expression analysis, the HA- and Flag-double tagged receptor was used. Data were assessed from a minimum of three independent experiments, each performed in triplicates and calculated to $100 \%$ of the positive control. Values represent mean + SEM. Significance was determined as NHA-MC4R + NIP compared to NHA-MC4R + RNF11. ${ }^{\star \star \star} p \leq 0.001$ (unpaired $t$-test, two tailed).

significantly reduced to $61 \%$ by co-expression with RNF11 (Figure 3A). Total receptor expression of both analyzed MCRs was not modified by co-expression with RNF11 (Figure 3B).

To analyze whether the decrease of GPCR expression and function in the presence of RNF11 is due to increased cell death, cell viability was investigated in HEK293 and COS-7 cells, corresponding to interaction, cAMP accumulation, reporter gene assays, or expression studies. As depicted in Figure 4, cell viability was not affected by RNF11 expression.

\section{RNF11 Has a Greater Impact on NFкB Signaling Reduction than $\alpha-\mathrm{MSH}$}

In addition to analysis of MC4R signaling and expression in interaction with RNF11, the influence of MC4R on RNF11 controlled action was also examined. Therefore, NFKB signaling was investigated by measuring NFKB-driven luciferase activity (Figure 5). NFKB luciferase activity was monitored in the absence (white columns) and presence (black columns) of $100 \mathrm{nM} \alpha-\mathrm{MSH}$ (Figure 5) and without (Figure 5A) or with (Figure 5B) additional NFkB stimulation by TNF $\alpha$. The first three column pairs of empty vector, NIP (rCHRM3), and RNF11 represent controls (Figure 5).

Data demonstrated clearly the reported NFKB inhibiting effect of RNF11 expressed alone $(19,20)$ compared with the empty vector alone, basal (Figure 5A), as well as under the $0.1 \mathrm{ng} / \mathrm{ml}$ TNF $\alpha$-stimulated condition (Figure 5B). Furthermore, basal $\mathrm{NF \kappa B}$ signaling (Figure 5A) was slightly yet significant reduced by MC3R and marginally enhanced by MC4R (MC3/4R in coexpression with NIP compared with NIP alone). Although alphaMSH had no additional decreasing effect on the basal NFKB state, RNF11 also inhibited NFKB signaling in co-expression of

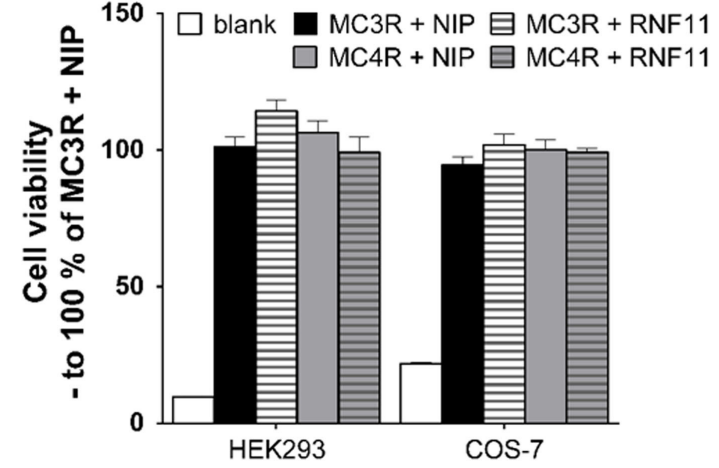

FIGURE 4 | Cell viability is not influenced by RNF11 expression. Cell viability during MC3/4R and NIP expression (black and gray columns) was compared with MC3/4R and RNF11 expression (striped columns) in the used cell systems. Data were assessed from a minimum of three independent experiments, each performed in triplicates and calculated to $100 \%$ control. Values represent mean + SEM.

MC3/4R almost completely (Figure 5A). Upon TNF $\alpha$ stimulation (Figure 5B), the reported NFKB inhibiting effect of $\alpha-\mathrm{MSH}$ on melanocortin receptors [(37) $\mathrm{MC} 3 / 4 \mathrm{R}$ co-expressed with NIP] was observed. A NFkB reduction of approximately $39 \%$ was documented (Figure 5B). However, RNF11 expression alone and in combination with $\mathrm{MC} 3 / 4 \mathrm{R}$ also resulted in the TNF $\alpha$ stimulated background to a stronger $\mathrm{NF \kappa B}$ abatement than $\mathrm{MSH}$. In all cases, NFKB signaling was reduced to approximately twofold over the basal empty vector by RNF11 (Figure 5B, reduction of 50-70\%). Interestingly, for MC3R/RNF11 co-expression, a slight synergistic effect of NFKB inhibition by RNF11 and 

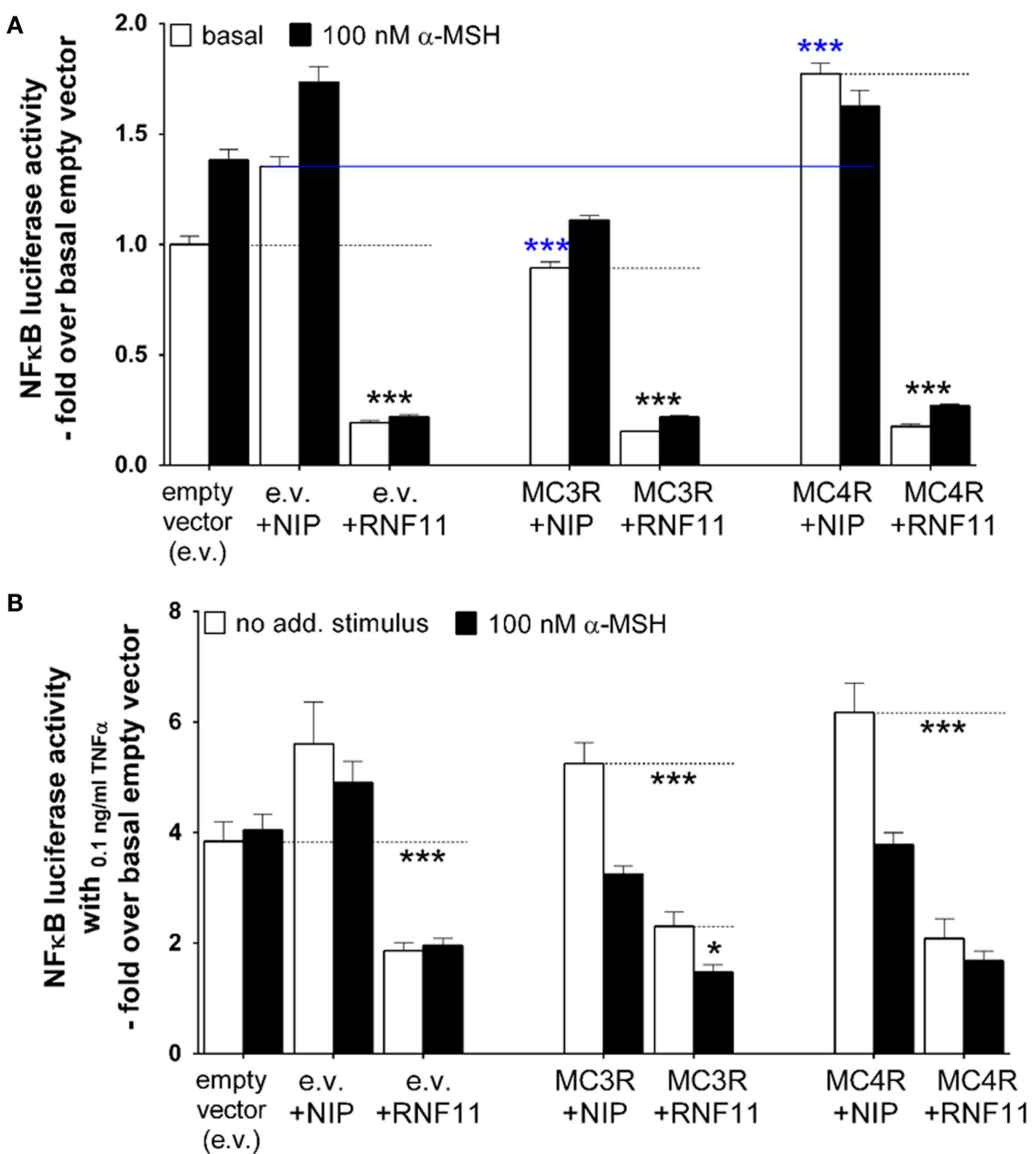

FIGURE 5 | RNF11 has stronger impact on decreasing NFкB signaling than stimulated MC3/4R. NFкB was measured using a luciferase reporter gene

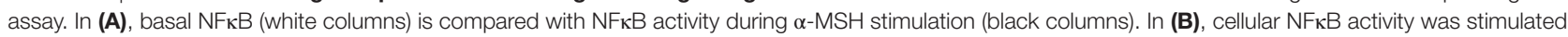
with $0.1 \mathrm{ng} / \mathrm{ml}$ TNF $\alpha$. In comparison, NFKB signaling in absence (white columns) and presence of $\alpha-\mathrm{MSH}$ (black columns). MC3R or MC4R in co-expression with the non-interactive protein [NIP, rCHRM3 (30)] is opposed to MC3/4R interactions with RNF11. The first three column pairs [empty vector (e.v.), e.v. + NIP, and e.v. + RNF11] served as controls. Data were assessed from a minimum of three independent experiments, each performed in triplicates, and calculated fold over the basal empty vector. Values represent mean + SEM. Significance determined by one-way ANOVA and Dunnett's test: basal e.v. compared with e.v. + RNF11 (A,B); basal e.v. + NIP compared with basal MC3/4R + NIP [(A), blue statistic]; basal MC3/4R + NIP to MC3/4R + RNF11 (A,B); Significance determined by unpaired $t$-test, two-tailed: $\alpha$-MSH effect on MC3/4R + NIP (B); $\alpha$-MSH effect on MC3R + RNF11 (B). ${ }^{*} p \leq 0.05 ;{ }^{* * *} p \leq 0.001$.

$\alpha$-MSH was observed, which indicates that basal NFkB signaling of MC3R + RNF11 is decreased compared with MC3R + NIP. A further decreasing effect in NFKB is obtained by $\alpha-\mathrm{MSH}$ stimulation in MC3R + RNF11 co-expression (Figure 5B).

\section{RNF11 Expression Trends toward an Increase after 3 Days of HFD}

The present data implies that RNF11 may play a role in the inhibition of MCR function during inflammation. Therefore, we analyzed whether hypothalamic NFкB inhibiting protein RNF11, as part of the A20 complex, is upregulated after 3 days of HFD, comparable to NFאB increasing inflammatory factors (1). As various genes are altered in expression after a short-term period of nutritional change (38), expression variation of six housekeeping genes were analyzed among standard diet and HFD-fed animals (see Materials and Methods). Only beta-actin was observed to be not affected by 3-day HFD and was used as reference gene in quantitative expression studies. Quantitative real-time PCR demonstrated Rnf11 expression in HFD-treated animals to be threefold higher than in mice that were fed a standard diet, although this was not significant (Figure 6).

Future studies should focus on whether methods other than BiFc can be used to analyze RNF11/MCR interaction. As Sandwich ELISA approaches failed to detect this interaction 


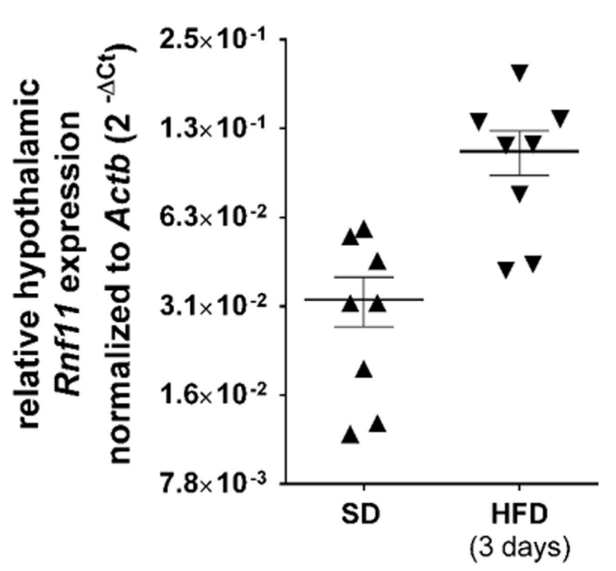

FIGURE 6 | Relative hypothalamic Rnf11 expression trends toward an increase following 3 days of HFD. Each group comprised of eight animals. qPCR of SD and HFD cDNA samples was performed in the same reaction plate. Gene expression of animals was analyzed in duplicates. All symbols display the mean of duplicates for one animal (detailed data can be found in Table S2 in Supplementary Material). For each diet group, the mean \pm SEM between the animals is depicted. Scale of $Y$-axis represents Log2. Reference gene, beta-actin (Actb); SD, standard diet; HFD, high-fat diet.

in the present study, we speculate that other proteins mediate the functional relevant RNF11/MCR interaction. Therefore, mass spectrometry may be applied whereby whole protein complexes are extracted in an initial step. In an additional step, RNF11 and MC3R or MC4R may be detected by tag-specific antibodies. Furthermore, more physiological cell lines such as hypothalamic cells are preferable for in vitro studies. In the present study, the hypothalamic neuronal cell lines N41, N39, and GT1-7 were analyzed; however, we were unable to obtain consistent results due to poor transfection efficiency or expression level. Still, in the conducted in vitro studies, RNF11 and MC3/4R are comparably overexpressed. Further studies have to reveal the physiological in vivo relevance of the results.

\section{DISCUSSION}

\section{RNF11 Influences MC3/4R Activity via Different Mechanisms}

Functional characterization of $\mathrm{MC} 3 / 4 \mathrm{R}$ revealed reduced signaling of these GPCRs due to co-expression with RNF11 (Figure 2). Furthermore, signaling inhibition was not restricted to the main MCR cAMP accumulation pathway, but was also constrained to the other analyzed pathways. Therefore, RNF11 appears to be a universal signaling blocking factor on MCRs. Further expression studies demonstrated that the RNF11 effect on MC3/4R is most likely generated by two different mechanisms.

(I) RNF11 can inhibit cell surface expression of a certain GPCR, as demonstrated in this study for MC4R, contributing to limited signaling capacity. Binding partners of RNF11 that have been identified so far suggest that RNF11 is likely to be involved in numerous signaling and trafficking processes (39). For example, RNF11 directly interacts with proteins of the ubiquitination machinery (ITCH, NEDD4, and UBC13) and with proteins mediating transport processes [e.g., GGA family of clathrin adaptors (40)]. RNF11 controls ubiquitination and thereby the function of GGA proteins, which have been shown to be involved in GPCR cell surface transport through direct interaction (41). Consistently, we demonstrated the ability of RNF11 to reduce MC4R cell surface expression, without changing total expression levels, which may be due to internalization or retaining of receptors in the endoplasmic reticulum and Golgi (Figures 3A,B).

(II) Analyses of the MC3R, which reside at the cell surface after RNF11 co-expression (Figure 3A), lead to the hypothesis that the association of RNF11 or RNF11-related protein complexes may inhibit GPCR activity by interfering with $\mathrm{G}$ protein coupling or $\mathrm{G}$ protein subunit release during activation.

These facts indicate specific actions of RNF11 on different GPCRs, resulting in the reduction of signaling capability. Several recognition sites on GPCRs, presented in different stages of receptor maturation, may determine favored RNF11 actions.

In addition to the RNF11 effect on MC3/4R function observed here, we also tested the impact of RNF11 on other hypothalamic GPCRs (Figure S1 in Supplementary Material). An interaction of RNF11 with the growth hormone secretagogues receptor (GHSR) and the G protein-coupled receptor 83 (GPR83) could be shown in addition to a signaling inhibition for both GPCRs through RNF11 co-expression. These data indicate a very consistent general action of RNF11 on GPCR function.

\section{RNF11 Function on Hypothalamic Melanocortin Receptors and Its Potential Role in Inflammation}

Chronic non-regulated inflammation is observed in the pathogenesis of obesity, diabetes, arteriosclerosis, Alzheimer's disease, and gout. Previously, numerous different factors such as acetylcholine (42), adenosine (43), or peptides, such as melanocortins $(44,45)$, have been identified to play a role in inflammation regulation. The targets of these factors are primarily GPCRs. For the first time, the present study indicates that RNF11 (as part of the A20 complex) represents a further factor that interacts with GPCRs. We speculate this interaction may be relevant in hypothalamic inflammation. NFkB, activated by $\mathrm{TNF} \alpha$, has been shown to lead to upregulation of A20 expression and to a higher interactivity of RNF11 for the assembly of the A20 complex, protecting the cell from constant NFKB signaling (20) in an auto-regulatory manner (Figure 7). By identifying RNF11 as an interaction partner of MC3/4R, we hypothesize that RNF11 potentially links hypothalamic inflammation to $\mathrm{MC} 3 / 4 \mathrm{R}$ signaling, a characteristic that has so far been attributed to leptin and insulin receptors. So far, Rnf11 knockout 


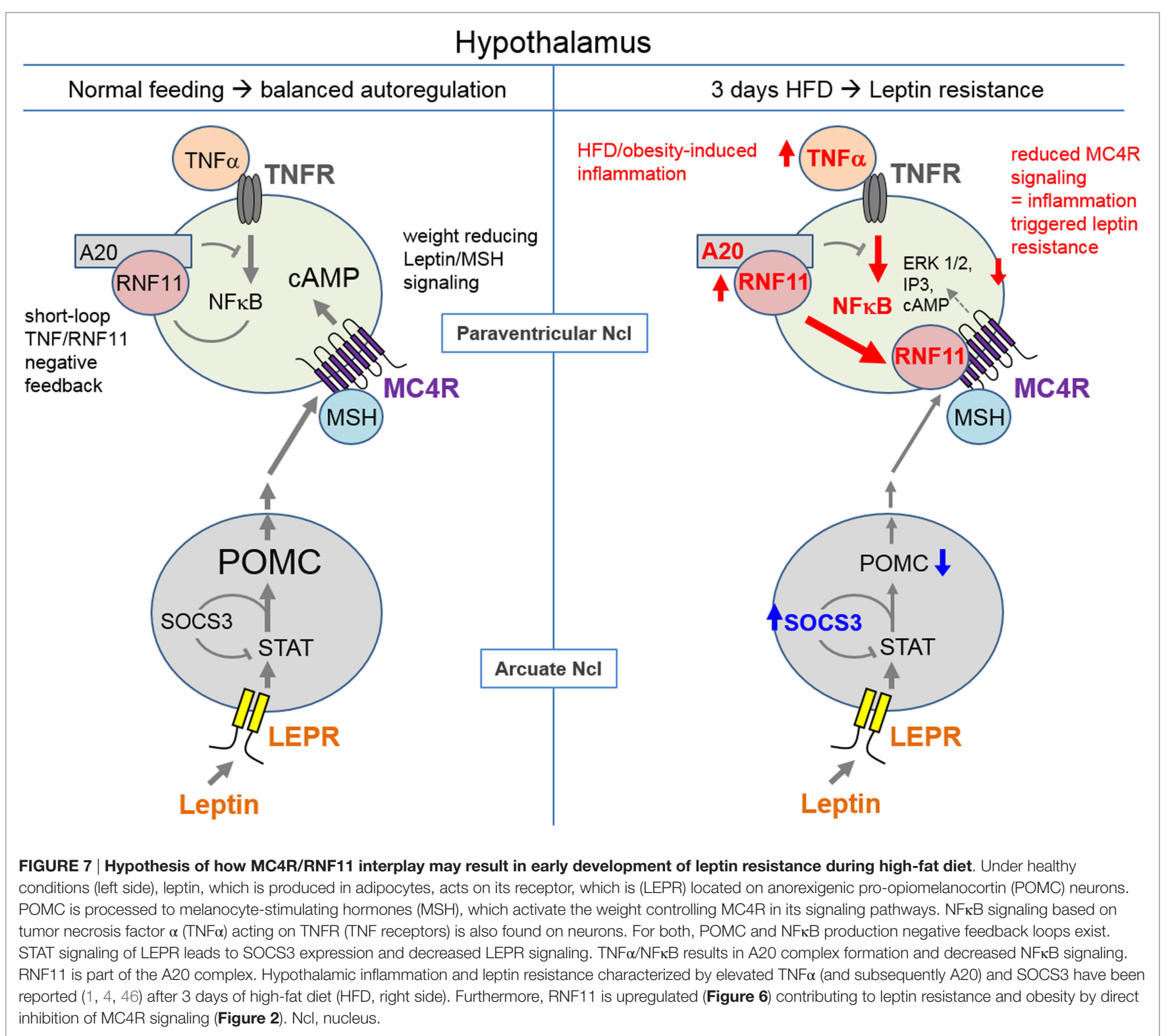

animals are not existent, probably due to lethality by the loss of the multifunctional protein.

\section{Altered Hypothalamic RNF11 Expression during the First Day of HFD May Contribute to Leptin Resistance and Obesity Manifestation}

Obesity and a HFD, even after 3 days (1), are associated with systemic low-grade inflammation and leptin resistance on the molecular signaling level. Circulating inflammatory cytokines such as TNFo, free fatty acids, and immune cells reach brain structures particularly at the hypothalamic level to provoke local inflammation [(47, 48), Figure 7]. Located at the third ventricle, and therefore exhibiting areas in which the blood-brain barrier is loosened, the hypothalamus, in particular the ARC, can be effectively reached by peripheral messengers. Peripheral inflammatory markers have been shown to activate microglia cells that produce local inflammatory cytokines such as TNF $\alpha$, in turn promoting a secondary hypothalamic inflammation process $(3,49)$. Hypothalamic inflammation, characterized by elevated TNF $\alpha$ or microglia activity, has been documented after a short-term HFD (1).

One prominent inflammatory pathway activated by TNF $\alpha$ is

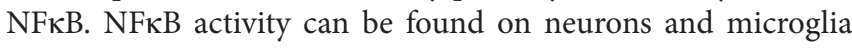
and is clearly associated with obesity [(50, 51), Figure 7]. Activation of $\mathrm{NF \kappa B}$ in microglia has been shown to lead to the degradation of, for example, dopaminergic neurons (52). Hypothalamic RNF11 appears to be upregulated in combination with A20 (Figure 7) to immediately counteract HFD-induced NFкB (Figure 6) and to provide protection particular to neurons. However, we hypothesize that higher RNF11 expression and 
activity increases the probability of MCR/RNF11 interaction, leading to desensitization of these GPCRs within 3 days of HFD (Figure 7). A decrease in the function of MC3R and particularly MC4R strongly contributes to the development of leptin resistance and obesity manifestation [(11, 13), Figure 7]. Moreover, the very early loss of MC4R function leads to increased food and caloric intake (11). Thus, RNF11 represent a further molecular detail how hypothalamic inflammation may trigger leptin resistance and obesity in the very early days of high caloric intake (Figure 7).

Our RNF11 expression studies were performed with complete hypothalami, including many different cell types (3). It would be of interest to observe whether significant RNF11 upregulation can be specifically found at the ARC and PVN neurons after 3 days of HFD.

Furthermore, hypothalamic expression and activity of RNF11 may potentially be regulated in a time-dependent manner. Constant RNF11 upregulation would block NFkB signaling and $\mathrm{MC} 3 / 4 \mathrm{R}$ or receptor resensitization, which may be of pathophysiological relevance.

In summary, RNF11 was presented as a new molecular link of how HFD exposure may result in functional melanocortin dysfunction. RNF11, which was observed to be marginally upregulated within the first 3 days of HFD feeding, inhibited MC3/4R function in in vitro studies. This lead to the hypothesis; that reduction of hypothalamic MC4R signaling, shortly after initiation of a HFD, may explain the early onset of functional leptin resistance, which contributes, in part, to further manifestations of obesity (Figure 7).

\section{REFERENCES}

1. Thaler JP, Yi CX, Schur EA, Guyenet SJ, Hwang BH, Dietrich MO, et al. Obesity is associated with hypothalamic injury in rodents and humans. J Clin Invest (2012) 122:153-62. doi:10.1172/JCI59660

2. Matsui Y, Tomaru U, Miyoshi A, Ito T, Fukaya S, Miyoshi H, et al. Overexpression of TNF-alpha converting enzyme promotes adipose tissue inflammation and fibrosis induced by high fat diet. Exp Mol Pathol (2014) 97:354-8. doi:10.1016/j.yexmp.2014.09.017

3. Kalin S, Heppner FL, Bechmann I, Prinz M, Tschop MH, Yi CX. Hypothalamic innate immune reaction in obesity. Nat Rev Endocrinol (2015) 11:339-51. doi:10.1038/nrendo.2015.48

4. Hotamisligil GS, Arner P, Caro JF, Atkinson RL, Spiegelman BM. Increased adipose tissue expression of tumor necrosis factor-alpha in human obesity and insulin resistance. J Clin Invest (1995) 95:2409-15. doi:10.1172/JCI117936

5. Olofsson LE, Unger EK, Cheung CC, Xu AW. Modulation of AgRP-neuronal function by SOCS3 as an initiating event in diet-induced hypothalamic leptin resistance. Proc Natl Acad Sci U S A (2013) 110:E697-706. doi:10.1073/ pnas. 1218284110

6. Koch CE, Lowe C, Pretz D, Steger J, Williams LM, Tups A. High-fat diet induces leptin resistance in leptin-deficient mice. J Neuroendocrinol (2014) 26:58-67. doi:10.1111/jne.12131

7. Posey KA, Clegg DJ, Printz RL, Byun J, Morton GJ, Vivekanandan-Giri A, et al. Hypothalamic proinflammatory lipid accumulation, inflammation, and insulin resistance in rats fed a high-fat diet. Am J Physiol Endocrinol Metab (2009) 296:E1003-12. doi:10.1152/ajpendo.90377.2008

8. Farooqi IS, O'Rahilly S. Mutations in ligands and receptors of the leptinmelanocortin pathway that lead to obesity. Nat Clin Pract Endocrinol Metab (2008) 4:569-77. doi:10.1038/ncpendmet0966

9. Siljee JE, Unmehopa UA, Kalsbeek A, Swaab DF, Fliers E, Alkemade A. Melanocortin 4 receptor distribution in the human hypothalamus. Eur J Endocrinol (2013) 168:361-9. doi:10.1530/EJE-12-0750

\section{AUTHOR CONTRIBUTIONS}

AM performed most of the experiments, analyzed the data, and wrote the manuscript. LN conducted the real-time PCR analysis and discussed the data. WJ and PW realized the feeding experiments, removed the hypothalami from animals and revised the work critically. C-XY, FM, JF, and GK helped in different experiments, contributed to the progression of the work and discussed the data. CG, AS, MT, AG, HK, and HB designed and supervised the project, enabled the experiments, analyzed and interpreted the data and wrote the manuscript.

\section{ACKNOWLEDGMENTS}

We would like to acknowledge the assistance of the BCRT Flow Cytometry Lab, particularly Desiree Kunkel (BCRT) for performing cell sorting experiments during the interaction screening.

\section{FUNDING}

This work wassupported by the Deutsche Forschungsgemeinschaft (DFG) BI 893/2-2, BI 893/5-2, BI 893/6-3, and BI 893/8-1, the Helmholtz Alliance "Imaging and Curing Environmental Metabolic Diseases" (ICEMED), WP12 and WP19, and by the Else Kröner-Fresenius-Stiftung (EKFS) project 2014_A114.

\section{SUPPLEMENTARY MATERIAL}

The Supplementary Material for this article can be found online at http://journal.frontiersin.org/article/10.3389/fendo.2016.00109

10. Cone RD. Anatomy and regulation of the central melanocortin system. Nat Neurosci (2005) 8:571-8. doi:10.1038/nn1455

11. Huszar D, Lynch CA, Fairchild-Huntress V, Dunmore JH, Fang Q, Berkemeier LR, et al. Targeted disruption of the melanocortin-4 receptor results in obesity in mice. Cell (1997) 88:131-41. doi:10.1016/S00928674(00)81865-6

12. Lubrano-Berthelier C, Cavazos M, Dubern B, Shapiro A, Stunff CL, Zhang S, et al. Molecular genetics of human obesity-associated MC4R mutations. Ann N Y Acad Sci (2003) 994:49-57. doi:10.1111/j.1749-6632.2003.tb03161.x

13. Farooqi IS, Yeo GS, O'Rahilly S. Binge eating as a phenotype of melanocortin 4 receptor gene mutations. N Engl J Med (2003) 349:606-9. doi:10.1056/ NEJM200308073490615

14. Chen AS, Marsh DJ, Trumbauer ME, Frazier EG, Guan XM, Yu H, et al. Inactivation of the mouse melanocortin-3 receptor results in increased fat mass and reduced lean body mass. Nat Genet (2000) 26:97-102. doi:10.1038/79254

15. Butler AA, Kesterson RA, Khong K, Cullen MJ, Pelleymounter MA, Dekoning $\mathrm{J}$, et al. A unique metabolic syndrome causes obesity in the melanocortin-3 receptor-deficient mouse. Endocrinology (2000) 141:3518-21. doi:10.1210/endo.141.9.7791

16. Watson SJ, Akil H, Richard CW III, Barchas JD. Evidence for two separate opiate peptide neuronal systems. Nature (1978) 275:226-8. doi:10.1038/ $275226 \mathrm{a} 0$

17. Yeo GS, Farooqi IS, Challis BG, Jackson RS, O'Rahilly S. The role of melanocortin signalling in the control of body weight: evidence from human and murine genetic models. QJM (2000) 93:7-14. doi:10.1093/qjmed/93.1.7

18. Santonico E, Belleudi F, Panni S, Torrisi MR, Cesareni G, Castagnoli L. Multiple modification and protein interaction signals drive the Ring finger protein 11 (RNF11) E3 ligase to the endosomal compartment. Oncogene (2010) 29:5604-18. doi:10.1038/onc.2010.294

19. Jacque E, Ley SC. RNF11, a new piece in the A20 puzzle. EMBO J (2009) 28:455-6. doi:10.1038/emboj.2009.18 
20. Shembade N, Parvatiyar K, Harhaj NS, Harhaj EW. The ubiquitin-editing enzyme A20 requires RNF11 to downregulate NF-kappaB signalling. EMBO J (2009) 28:513-22. doi:10.1038/emboj.2008.285

21. Nyfeler B, Reiterer V, Wendeler MW, Stefan E, Zhang B, Michnick SW, et al. Identification of ERGIC-53 as an intracellular transport receptor of alpha1antitrypsin. J Cell Biol (2008) 180:705-12. doi:10.1083/jcb.200709100

22. Muller A, Kleinau G, Piechowski CL, Muller TD, Finan B, Pratzka J, et al. G-protein coupled receptor 83 (GPR83) signaling determined by constitutive and zinc(II)-induced activity. PLoS One (2013) 8:e53347. doi:10.1371/journal. pone. 0053347

23. Rediger A, Tarnow P, Bickenbach A, Schaefer M, Krude H, Gruters A, et al. Heterodimerization of hypothalamic G-protein-coupled receptors involved in weight regulation. Obes Facts (2009) 2:80-6. doi:10.1159/000209862

24. Muller TD, Muller A, Yi CX, Habegger KM, Meyer CW, Gaylinn BD, et al. The orphan receptor Gpr83 regulates systemic energy metabolism via ghrelin-dependent and ghrelin-independent mechanisms. Nat Commun (2013) 4:1968. doi: $10.1038 /$ ncomms 2968

25. Schulz A, Grosse R, Schultz G, Gudermann T, Schoneberg T. Structural implication for receptor oligomerization from functional reconstitution studies of mutant V2 vasopressin receptors. J Biol Chem (2000) 275:2381-9. doi:10.1074/jbc.275.4.2381

26. Rediger A, Piechowski CL, Yi CX, Tarnow P, Strotmann R, Gruters A, et al. Mutually opposite signal modulation by hypothalamic heterodimerization of ghrelin and melanocortin-3 receptors. J Biol Chem (2011) 286:39623-31. doi:10.1074/jbc.M111.287607

27. Chan LF, Webb TR, Chung TT, Meimaridou E, Cooray SN, Guasti L, et al. MRAP and MRAP2 are bidirectional regulators of the melanocortin receptor family. Proc Natl Acad Sci U S A (2009) 106:6146-51. doi:10.1073/ pnas. 0809918106

28. Sudol M, Chen HI, Bougeret C, Einbond A, Bork P. Characterization of a novel protein-binding module - the WW domain. FEBS Lett (1995) 369:67-71. doi:10.1016/0014-5793(95)00550-S

29. Kitching R, Wong MJ, Koehler D, Burger AM, Landberg G, Gish G, et al. The RING-H2 protein RNF11 is differentially expressed in breast tumours and interacts with HECT-type E3 ligases. Biochim Biophys Acta (2003) 1639:104-12. doi:10.1016/j.bbadis.2003.07.001

30. Elsner A, Tarnow P, Schaefer M, Ambrugger P, Krude H, Gruters A, et al. MC4R oligomerizes independently of extracellular cysteine residues. Peptides (2006) 27:372-9. doi:10.1016/j.peptides.2005.02.027

31. Vongs A, Lynn NM, Rosenblum CI. Activation of MAP kinase by MC4-R through PI3 kinase. Regul Pept (2004) 120:113-8. doi:10.1016/j. regpep.2004.02.018

32. He S, Tao YX. Defect in MAPK signaling as a cause for monogenic obesity caused by inactivating mutations in the melanocortin- 4 receptor gene. Int J Biol Sci (2014) 10:1128-37. doi:10.7150/ijbs.10359

33. Minokoshi Y, Alquier T, Furukawa N, Kim YB, Lee A, Xue B, et al. AMP-kinase regulates food intake by responding to hormonal and nutrient signals in the hypothalamus. Nature (2004) 428:569-74. doi:10.1038/nature02440

34. Chai B, Li JY, Zhang W, Wang H, Mulholland MW. Melanocortin-4 receptor activation inhibits c-Jun $\mathrm{N}$-terminal kinase activity and promotes insulin signaling. Peptides (2009) 30:1098-104. doi:10.1016/j.peptides.2009.03.006

35. Chai B, Li JY, Zhang W, Newman E, Ammori J, Mulholland MW. Melanocortin-4 receptor-mediated inhibition of apoptosis in immortalized hypothalamic neurons via mitogen-activated protein kinase. Peptides (2006) 27:2846-57. doi:10.1016/j.peptides.2006.05.005

36. Newman EA, Chai BX, Zhang W, Li JY, Ammori JB, Mulholland MW. Activation of the melanocortin-4 receptor mobilizes intracellular free calcium in immortalized hypothalamic neurons. J Surg Res (2006) 132:201-7. doi:10.1016/j.jss.2006.02.003

37. Lee SN, Ryu JH, Joo JH, Choi YH, Lee HJ, Kim YJ, et al. alpha-Melanocyte-stimulating hormone inhibits tumor necrosis factor alpha-stimulated MUC5AC expression in human nasal epithelial cells. Am J Respir Cell Mol Biol (2011) 44:716-24. doi:10.1165/rcmb.2009-04200C
38. Sparks LM, Xie H, Koza RA, Mynatt R, Hulver MW, Bray GA, et al. A high-fat diet coordinately downregulates genes required for mitochondrial oxidative phosphorylation in skeletal muscle. Diabetes (2005) 54:1926-33. doi:10.2337/diabetes.54.7.1926

39. Azmi P, Seth A. RNF11 is a multifunctional modulator of growth factor receptor signalling and transcriptional regulation. Eur J Cancer (2005) 41:2549-60. doi:10.1016/j.ejca.2005.08.020

40. Santonico E, Mattioni A, Panni S, Belleudi F, Mattei M, Torrisi MR, et al. RNF11 is a GGA protein cargo and acts as a molecular adaptor for GGA3 ubiquitination mediated by Itch. Oncogene (2015) 34:3377-90. doi:10.1038/ onc.2014.256

41. Zhang M, Davis JE, Li C, Gao J, Huang W, Lambert NA, et al. GGA3 interacts with a G protein-coupled receptor and modulates its cell surface export. Mol Cell Biol (2016) 36:1152-63. doi:10.1128/MCB.00009-16

42. The FO, Boeckxstaens GE, Snoek SA, Cash JL, Bennink R, Larosa GJ, et al. Activation of the cholinergic anti-inflammatory pathway ameliorates postoperative ileus in mice. Gastroenterology (2007) 133:1219-28. doi:10.1053/j. gastro.2007.07.022

43. Ehrentraut H, Clambey ET, McNamee EN, Brodsky KS, Ehrentraut SF, Poth JM, et al. CD73+ regulatory T cells contribute to adenosine-mediated resolution of acute lung injury. FASEB J (2013) 27:2207-19. doi:10.1096/ f. $12-225201$

44. La M, Tailor A, D’Amico M, Flower RJ, Perretti M. Analysis of the protection afforded by annexin 1 in ischaemia-reperfusion injury: focus on neutrophil recruitment. Eur J Pharmacol (2001) 429:263-78. doi:10.1016/ S0014-2999(01)01325-5

45. Getting SJ, Gibbs L, Clark AJ, Flower RJ, Perretti M. POMC gene-derived peptides activate melanocortin type 3 receptor on murine macrophages, suppress cytokine release, and inhibit neutrophil migration in acute experimental inflammation. J Immunol (1999) 162:7446-53.

46. Guo S, Messmer-Blust AF, Wu J, Song X, Philbrick MJ, Shie JL, et al. Role of A20 in cIAP-2 protection against tumor necrosis factor alpha (TNF-alpha)mediated apoptosis in endothelial cells. Int J Mol Sci (2014) 15:3816-33. doi:10.3390/ijms15033816

47. Sternberg EM. Neural-immune interactions in health and disease. J Clin Invest (1997) 100:2641-7. doi:10.1172/JCI119807

48. Perry SW, Dewhurst S, Bellizzi MJ, Gelbard HA. Tumor necrosis factor-alpha in normal and diseased brain: conflicting effects via intraneuronal receptor crosstalk? J Neurovirol (2002) 8:611-24. doi:10.1080/13550280290101021

49. Perry VH, Teeling J. Microglia and macrophages of the central nervous system: the contribution of microglia priming and systemic inflammation to chronic neurodegeneration. Semin Immunopathol (2013) 35:601-12. doi:10.1007/ s00281-013-0382-8

50. Tang Y, Cai D. Hypothalamic inflammation and GnRH in aging development. Cell Cycle (2013) 12:2711-2. doi:10.4161/cc.26054

51. Tzanavari T, Giannogonas P, Karalis KP. TNF-alpha and obesity. Curr Dir Autoimmun (2010) 11:145-56. doi:10.1159/000289203

52. Lee JK, Chung J, McAlpine FE, Tansey MG. Regulator of G-protein signaling-10 negatively regulates NF-kappaB in microglia and neuroprotects dopaminergic neurons in hemiparkinsonian rats. J Neurosci (2011) 31:11879-88. doi:10.1523/JNEUROSCI.1002-11.2011

Conflict of Interest Statement: The authors declare that the research was conducted in the absence of any commercial or financial relationships that could be construed as a potential conflict of interest.

Copyright $\odot 2016$ Müller, Niederstadt, Jonas, Yi, Meyer, Wiedmer, Fischer, Grötzinger, Schürmann, Tschöp, Kleinau, Grüters, Krude and Biebermann. This is an open-access article distributed under the terms of the Creative Commons Attribution License (CC BY). The use, distribution or reproduction in other forums is permitted, provided the original author(s) or licensor are credited and that the original publication in this journal is cited, in accordance with accepted academic practice. No use, distribution or reproduction is permitted which does not comply with these terms. 